\title{
AIM Platform: A Novel Nano Artificial Antigen-Presenting Cell-Based Clinical System Designed to Consistently Produce Multi-Antigen-Specific T-Cell Products with Potent and Durable Anti-Tumor Properties
}

\author{
Lauren Suarez Ruipeng Wang Scott Carmer Daniel Bednarik Han Myint \\ Kristi Jones Mathias Oelke \\ NexImmune, Gaithersburg, MD, USA
}

\begin{abstract}
Keywords
Acute myeloid leukemia - Multiple myeloma · Artificial antigen-presenting cell · Adoptive cell therapy · CD8 · Human · Clinical trial · T cells, T-cell manufacturing · Dendritic cells · Prodigy, CliniMACS · GMP · Nano, nanoparticle
\end{abstract}

\begin{abstract}
Over the last decade, tremendous progress has been made in the field of adoptive cell therapy. The two prevailing modalities include endogenous non-engineered approaches and genetically engineered T-cell approaches. Endogenous non-engineered approaches include dendritic cell-based systems and tumor-infiltrating lymphocytes (TIL) that are used to produce multi-antigen-specific T-cell products. Genetically engineered approaches, such as T-cell receptor engineered cells and chimeric antigen receptor T cells are used to produce single antigen-specific T-cell products. It is noted by the authors that there are alternative methods to sort for antigen-specific $T$ cells such as peptide multimer sorting or cytokine secretion assay-based sorting, both of which are potentially challenging for broad development and commercialization. In this review, we are focusing on a novel nanoparticle technology that generates a non-engineered product from the endogenous T-cell repertoire. The most common approaches for ex vivo activation and expansion of
\end{abstract}

endogenous, non-genetically engineered cell therapy products rely on dendritic cell-based systems or IL-2 expanded TIL. Hurdles remain in developing efficient, consistent, controlled processes; thus, these processes still have limited access to broad patient populations. Here, we describe a novel approach to produce cellular therapies at clinical scale, using proprietary nanoparticles combined with a proprietary manufacturing process to enrich and expand antigen-specific $\mathrm{CD}^{+}$T-cell products with consistent purity, identity, and composition required for effective and durable anti-tumor response.

๑) 2020 S. Karger AG, Basel

\section{Introduction}

Antigen-presenting cells (APCs) are immune cells that play major roles in processing and presenting antigens for recognition by T lymphocytes. Dendritic cells (DCs) are the most potent APCs for activating and directing the function of antigen-specific $\mathrm{T}$ cells required to kill diseased or infected cells. Due to the nature of tissue residency of mature DCs capable of effective antigen presentation, it can be difficult to obtain the numbers of DCs required $\mathrm{T}$-cell stimulation from peripheral blood [1]. Monocytes, which are the natural progenitors of DCs, can be more easily isolated from peripheral blood mononu- 


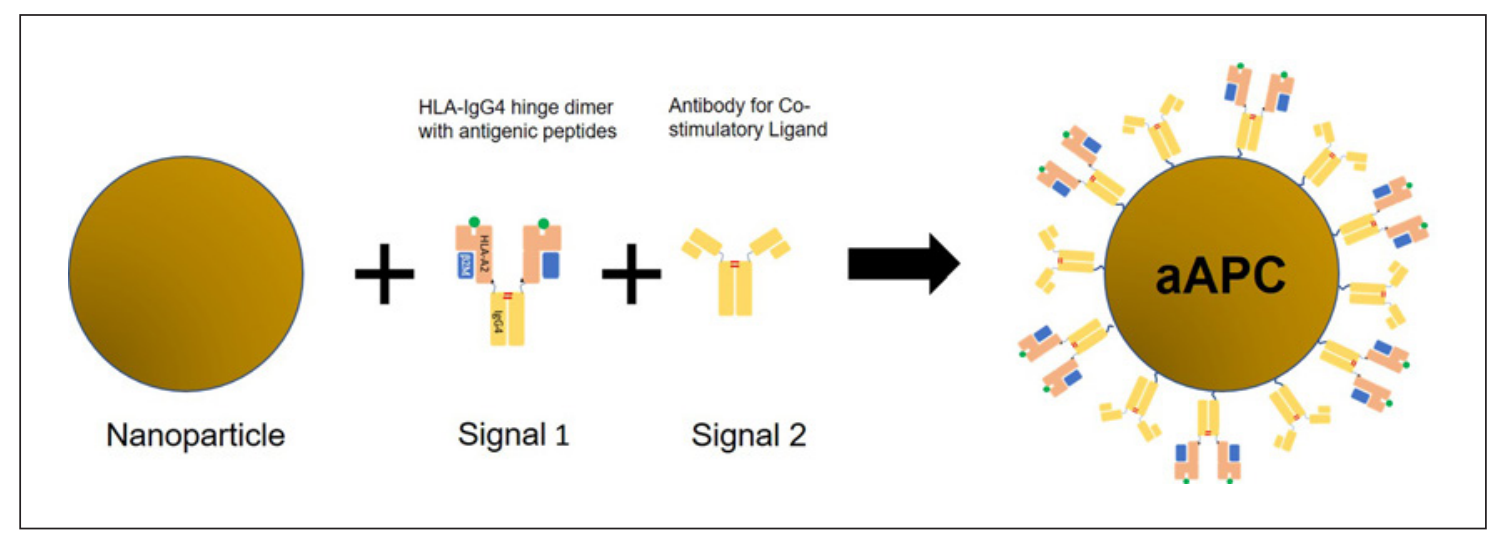

Fig. 1. AIM-np consist of a superparamagnetic iron-oxide nanoparticle, to which humanized HLA-A2-IgG4 hinge dimer (signal 1) and humanized anti-CD28 antibody (signal 2) molecules are covalently linked. AIM-np can be loaded with an HLA-A*02:01-restricted peptide of choice (shown in green) and lead to the activation and proliferation of antigen-specific $\mathrm{CD} 8^{+} \mathrm{T}$ cells. aAPC, artificial antigen-presenting cell.

clear cells and differentiated into mature DCs [2]. While isolation and in vitro differentiation of progenitor cells into functional DCs for T-cell stimulation is feasible, the progenitor differentiation process is lengthy, expensive, and results in a high degree of variability with respect to numbers and quality of APCs produced. In cancer patients, both APCs and their progenitor cells are often compromised or impaired, which can further challenge the reproducibility of the process [3-5]. In addition, controlling specific DC antigen presentation to T cells is not biologically feasible. Despite clinical progress, these fundamental hurdles remain as limitations for all APC-based systems. To overcome these limitations, we have developed a rationally designed nanoparticle, termed AIM-np (Artificial Immune Modulation nanoparticle), that functions as a nano-sized synthetic APC, which can be constructed to deliver precise instructions directly to specific populations of $\mathrm{CD} 8^{+} \mathrm{T}$ cells. AIM-np can be reproducibly manufactured and easily customized for different epitope targets or T-cell function.

\section{The AIM Platform}

The Artificial Immune Modulation (AIM) technology platform is comprised of two key components, AIM nanoparticles (AIM-np) that function as 'artificial APCs' and a proprietary cell therapy manufacturing process termed Enrichment + Expansion (E+E) [6]. When combined, the AIM-np and $\mathrm{E}+\mathrm{E}$ process produce a cell therapy with highly reproducible product attributes that have been optimized for deep and durable anti-tumor clinical responses.

\section{AIM Nanoparticle}

AIM-np (shown in Fig. 1) act as nano-sized synthetic APCs and are designed to direct the natural immune function of antigen-specific $\mathrm{CD} 8^{+} \mathrm{T}$ cells. AIM-np consist of a superparamagnetic iron-oxide nanoparticle core decorated with two humanized signaling proteins. HLAA2-IgG4 hinge dimer molecules are conjugated to the core nanoparticle to deliver signal 1 (antigen presentation), together with humanized anti-CD28 antibodies, which deliver signal 2 (co-stimulation). Subsequently, the AIM-np acts as a synthetic APC by directly engaging targeted $\mathrm{T}$ cells through naturally occurring signaling mechanisms. Signal 1 is delivered by a peptide-loaded HLA class I (e.g., HLA-A*02:01) dimerized fusion protein that presents antigen peptides to cognate $\mathrm{T}$-cell receptors; signal 2 is delivered by a monoclonal antibody to the CD28 receptor delivering the co-stimulatory signal, also called "danger signal," to induce antigen-specific T-cell activation and proliferation. While the AIM-np employed in ongoing phase $1 / 2$ clinical trials use the HLA subtype, HLA-*A02:01, other HLA class I subtypes are under development to be used interchangeably according to the individual patients being treated, thereby creating an "off-the-shelf" antigen presentation modality.

For clinical use, aliquots of AIM-np are loaded with individual HLA-restricted disease-relevant peptides, which can be derived from either surface or intracellular proteins that are overexpressed on target (diseased) cells. Multiple aliquots of individually loaded AIM-np can be combined to produce $\mathrm{T}$-cell products capable of targeting multiple disease-relevant antigens. The peptide-loaded AIM-np effectively engage antigen-specific T cells without the need of native APCs. AIM-np can be further customized via peptide selection to target a variety of tumor types. 


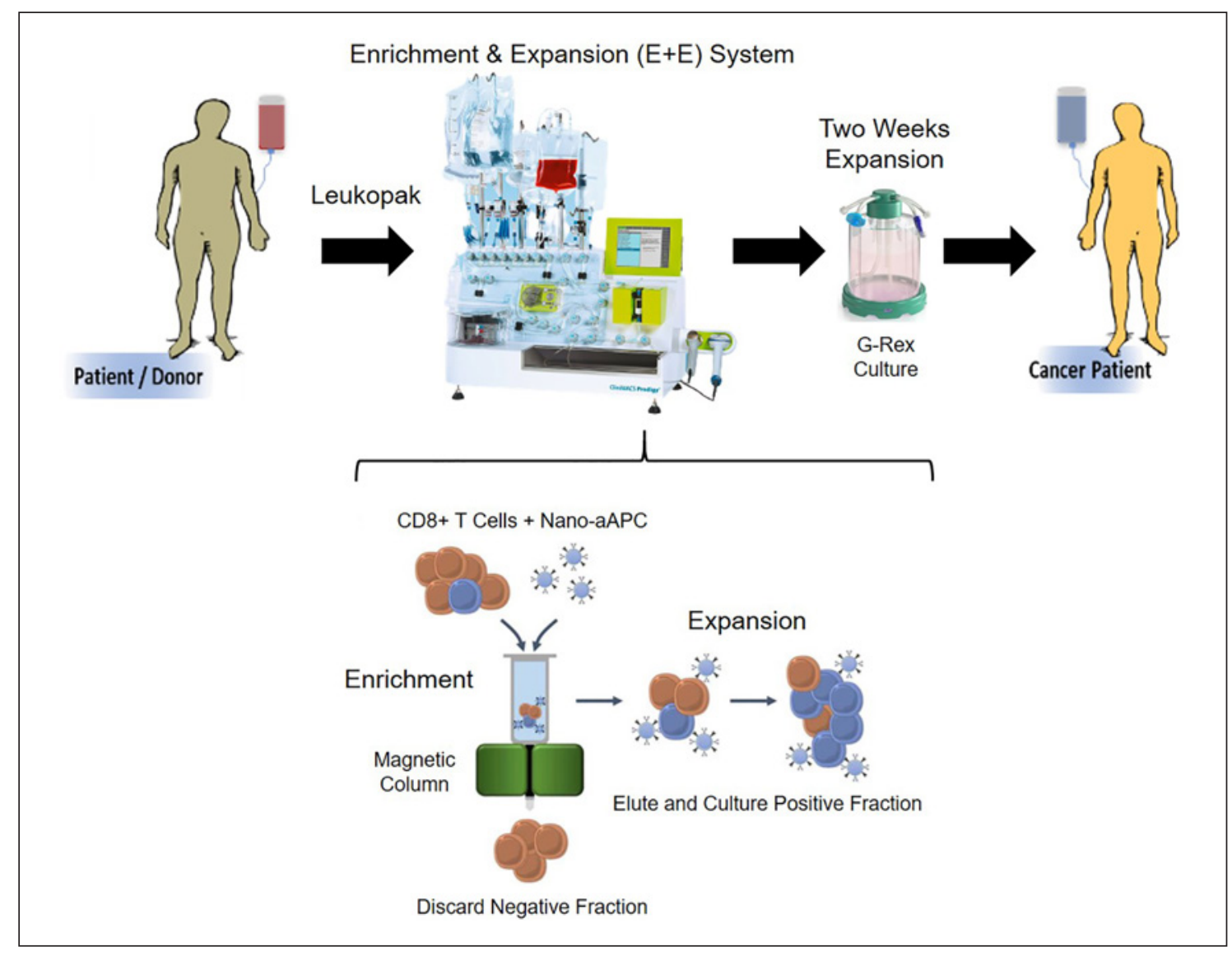

Fig. 2. Enrichment and Expansion of antigen-specific $\mathrm{CD}^{+} \mathrm{T}$ cells. Leukapheresis material is collected from human donors and put into a CliniMacs-Prodigy ${ }^{\circledR}$ cell expansion system (Miltenyi Biotec). Magnetic separation is used to deplete $\mathrm{CD} 4^{+} \mathrm{T}$ cells and to enrich for antigen-specific $\mathrm{CD} 8^{+} \mathrm{T}$ cells using the AIM-np. The enriched cell suspension is placed in G-Rex expansion flasks in combination with a proprietary cytokine mix. Cultures are expanded for 2 weeks. Using this process allows for the consistent generation of clinically relevant numbers of antigen-specific $\mathrm{CD}^{+} \mathrm{T}$ cells (shown as blue cells). Portions of cartoon adapted from Perica et al. [6].

\section{E+E Manufacturing Process}

Reproducible manufacturing processes for DC-based T-cell therapies remain a challenge, and manufacturing scaling issues limit access to broader patient populations. While early clinical trials have demonstrated encouraging results in small numbers of cancer patients [7], production of these therapies has traditionally been hampered due to a lengthy, difficult-to-control, and costly manufacturing process that yields a highly variable T-cell product. In addition, these endogenous DC-based approaches are not easily scalable for widespread manufacture. Finally, controlling precise DC antigen processing and presentation to $\mathrm{T}$ cells is not possible. Replacing DCs with the AIM-np provides direct engagement with cognate $\mathrm{T}$ cells, which when combined with a T-cell enrichment and expansion process $(\mathrm{E}+\mathrm{E})$ represents a novel approach designed to overcome these hurdles. Clinical manufacturing is conducted in the CliniMACS Prodigy, which has been customized to run the $\mathrm{E}+\mathrm{E}$ process in a fully en- closed, semi-automated cGMP system, and therefore, the manufacturing system is highly controllable, reproducible, and scalable (shown in Fig. 2).

Using this system, antigen-specific $\mathrm{CD}^{+} \mathrm{T}$ cells can be enriched from either a donor-derived or patient-derived leukopak in a sterile, reliable fashion and then expanded to therapeutically relevant $\mathrm{T}$-cell numbers (shown in Fig. 3A). The first step in the E+E process involves the depletion of $\mathrm{CD} 4^{+} \mathrm{T}$ cells from the leukopak via magnetic separation. Depletion of these cells helps ensure that there are no T regulatory (Treg) cells in culture or the final product. Removal of Treg cells has been demonstrated to enhance anti-tumor effect [8]. After $\mathrm{CD}^{+}$cell depletion, the remaining cells are enriched for $\mathrm{CD}^{+} \mathrm{T}$-cell populations specific to the antigen peptides of interest. This is accomplished by step 2 in the process, where the cells are incubated with a cocktail of peptide-loaded AIM$\mathrm{np}$, containing multiple AIM-np aliquots individually loaded with different tumor associated antigen peptides. 
A

AML E+E total T cells (Day 14)

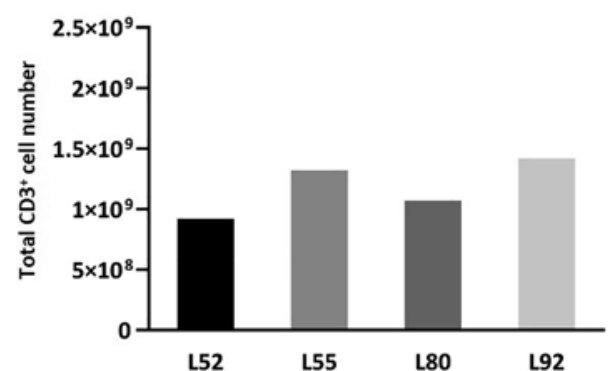

C

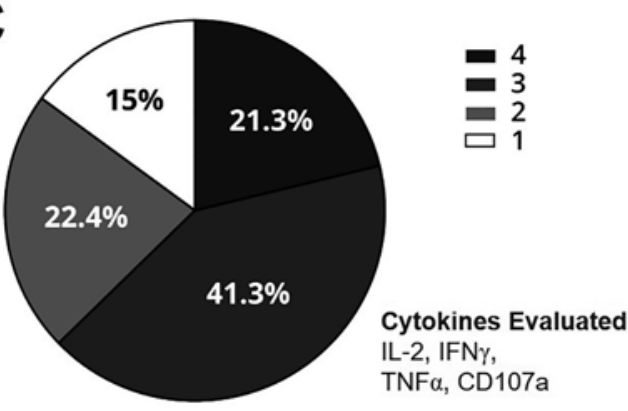

$62 \%$ of $A M L$ specific T cells demonstrate 3-4 effector functions upon non-specific stimulation
B AML antigen specific T cells (Day 14)

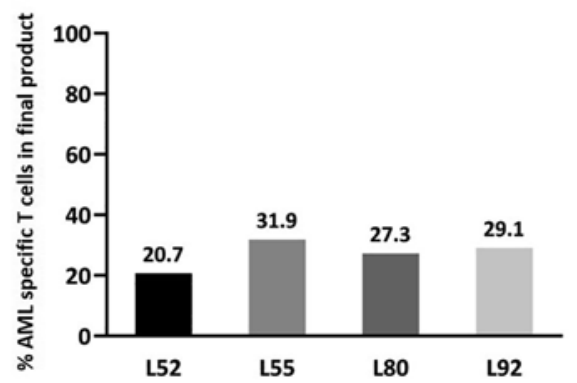

D AML E+E \% T cells with effector function

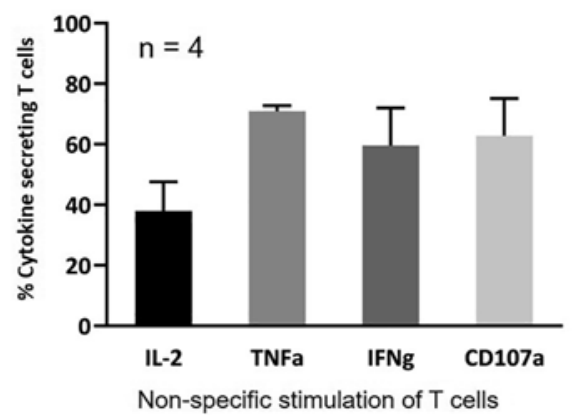

Fig. 3. Characteristics of AML T cells produced by Enrichment and Expansion process using peptide cocktail derived from WT1, PRAME, and cyclin A1. A Our manufacturing process produces a T-cell product at therapeutically relevant cell numbers. B Total antigen specificity $(n=4)$ is upwards of 20\%. L52, L55, L80, and L92 refer to individual leukopak experiments $(\mathbf{A}$ and $\mathbf{B})$. The $\mathrm{T}$ cells in the final product are polyfunctional and display multiple effector functions upon non-specific stimulation (C and D).

The individual AIM-np identify and engage their cognate $\mathrm{T}$-cell receptors from the endogenous $\mathrm{CD} 8^{+} \mathrm{T}$-cell repertoire. $\mathrm{CD} 8^{+} \mathrm{T}$ cells bound to the AIM-np are separated using the magnetic column, while all other cells including monocytes, B cells, NK cells, and non-antigen-specific $\mathrm{CD}^{+} \mathrm{T}$ cells are discarded. In step 3 of the process, the enriched $\mathrm{CD}^{+} \mathrm{T}$ cells are eluted from the magnetic column, together with any unbound AIM-np, and transferred to a G-Rex culture system (Wilson-Wolf Manufacturing) for a subsequent 14-day expansion period as shown in Figure 2. Throughout the expansion process, cells are fed with a cytokine cocktail optimized to expand $\mathrm{T}$ cells and to yield a product that is composed of both antigen-specific and high-quality memory $\mathrm{CD} 8^{+} \mathrm{T}$ cells. Using anti-CD28 monoclonal antibody as a co-stimulatory molecule supports engagement and expansion of $\mathrm{T}$ cells from the naïve repertoire, which, in turn, enables the ability to control the in vivo $\mathrm{T}$-cell differentiation process in a way that consistently produces less differentiated $\mathrm{T}$ cells. These less differentiated T-cell subtypes possess an- ti-tumor, proliferative and self-renewal properties $[9,10]$. This is meaningfully contrasted with the more differentiated T-cell subtypes produced from the multiple rounds of re-stimulation or manipulation required with DCbased expansion procedures $[7,11,12]$.

The AIM technology has been optimized for clinical use in the following ways:

1. Sensitivity: AIM-np have the ability to target, activate, and expand antigen-specific $T$ cells from very-low-frequency populations and are designed to engage $\mathrm{T}$ cells from the naïve and memory repertoires through natural signaling mechanisms.

2. Process control and reproducibility: Consistent production of T-cell products that are highly pure and contain antigen-specific populations of naturally occurring $\mathrm{CD}^{+} \mathrm{T}$ cells.

3. Product composition: The incorporation of the AIM$\mathrm{np}$ as part of the proprietary E+E manufacturing process produces $T$ cells with a unique and powerful combination of potency, target-specific activity, and long- 
Table 1. Clinical benefits of NexImmune T-cell product

\begin{tabular}{|c|c|}
\hline Antigen-tumor potency & $\begin{array}{l}\text { - Each product contains populations of } \mathrm{T} \text { cells directed against multiple tumor-specific targets, } \\
\text { including cell surface and endogenous antigen peptides } \\
\text { - AIM-expanded T cells are polyclonal and contain a broad repertoire of both high- and low-affinity } \\
\text { T-cell receptors } \\
\text { - After target engagement, AIM-expanded T cells initiate a polyfunctional cytokine reaction capable of } \\
\text { recruiting CD4 } 4^{+} \text {T cells to generate a robust T cell-mediated immune response }\end{array}$ \\
\hline Long-term persistence & $\begin{array}{l}\text { - Each product contains the T-cell subtypes essential to anti-tumor activity, proliferation, and self- } \\
\text { renewal }\end{array}$ \\
\hline Safety & $\begin{array}{l}\text { - Because AIM-expanded T cells are generated from the natural repertoire and are not genetically } \\
\text { engineered, they can effectively distinguish diseased cells from healthy cells and exhibit highly target- } \\
\text { specific killing; as a result, these T cells are not expected to demonstrate the life-threatening toxicities } \\
\text { commonly associated with genetically modified T-cell therapies }\end{array}$ \\
\hline
\end{tabular}

term persistence. Translating this aspect into the company's current cancer product achieves clinical benefits outlined in Table 1.

4. AIM-np construct: The "Lego-like" nature of the AIM platform enables rapid and straightforward construction of a variety of synthetic DCs (AIM-np) that can be designed to activate or suppress antigen-specific $\mathrm{T}$ cell function, providing utility across cancer, auto-immune, and infectious diseases.

\section{In vitro Characterization of AIM-expanded T-Cell Products}

Despite demonstrations of safety and efficacy in earlystage clinical trials, there are significant limitations attributed to current DC-based adoptive T-cell therapy strategies, including poor T-cell engraftment and persistence, lengthy manufacturing timelines, and diminished therapeutic effectiveness [9]. In the following section, we describe the most important characteristics of AIM-expanded T-cell products designed to overcome these limitations through in vitro characterizing of the following critical cell therapy attributes: T cell specificity, phenotype, and functionality.

Antigen Specificity of AIM-Expanded T-Cell Products

A primary mechanism of tumor escape is "target downregulation." This occurs when a tumor cell is under immune pressure and downregulates or stops expressing the specific antigen being attacked by the immune system. In the case of chimeric antigen receptor T-cell therapy, data has shown that malignant B cells have the ability to downregulate the expression of CD19 surface proteins as a way to escape recognition by the genetically engineered T cells $[13,14]$. Cellular therapies that can direct populations of $\mathrm{T}$ cells against multiple tumor-specific antigen targets represent a promis- ing approach to address this primary escape mechanism.

As described, our AIM-np based E+E process targets multiple peptides from different tumor antigens simultaneously to produce multi-antigen-specific T-cell products. In the examples shown in Figure 3B, we have used an AIM-np cocktail that was loaded with five peptides from multiple acute myeloid leukemia (AML) antigens to minimize the risk for immune escape [15-17]. Using five antigen peptides also increases the likelihood that at least one of these antigens is highly expressed in any given patient's tumor. The final T-cell product is composed of (on average) more than $20 \%$ antigen-specific cells across all antigens (Fig. 3B). In a separate study comparing specificity, phenotype, and functionality of T-cell products, Ichikawa et al. [18] have shown that the AIM-np based E+E process generates Mart1-specific T-cell products from both healthy donors and melanoma patients that are far superior to T-cell products that were generated with peptide-loaded autologous DCs. As antigen-specific T cells are enriched from the endogenous $\mathrm{T}$-cell repertoire, donor-to-donor variability will affect final composition of the product. However, the AIM E+E system produces similar results using healthy donor or patient cells. Finally, the number of antigens and peptide targets can be geared up or down for future development.

\section{T-Cell Phenotype}

Ideal $\mathrm{T}$-cell therapies contain $\mathrm{T}$-cell subtypes that combine the anti-tumor potency and long-term persistence required to deliver both deep and durable clinical responses. T-cell subtypes that are the primary drivers of tumor cell killing reside in the $\mathrm{T}$ effector memory $\left(\mathrm{T}_{\mathrm{em}}\right)$ compartment. The subtypes that are primarily responsible for T-cell proliferation and immunologic memory are found in the $\mathrm{T}$ central memory $\left(\mathrm{T}_{\mathrm{cm}}\right)$ compartment, while subtypes required for self-renewal and long-term longev- 


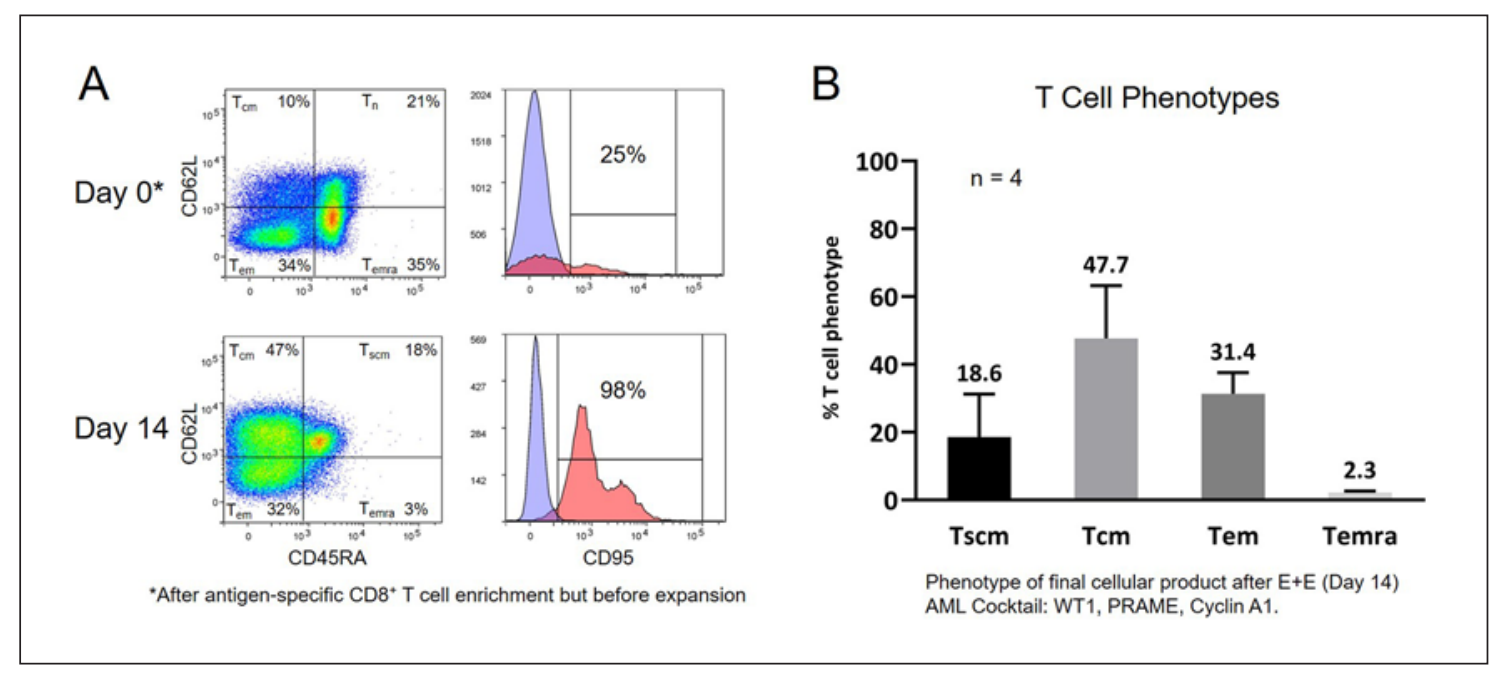

Fig. 4. The representative data shows over $95 \%$ cells in our final T-cell product are memory phenotype. A A representative breakdown of memory subtypes defined by CD62L and CD45RA as indicated in the dot plot. T naïve cells and $\mathrm{T}_{\mathrm{scm}}$ cells are further distinguished by CD95 expression as shown in the histograms. The histogram is an analysis of the CD62L $\mathrm{L}^{+}, \mathrm{CD} 45 \mathrm{RA}^{+}$cells. B Overview of memory subtype distribution across 4 products. T stem cell memory $\left(\mathrm{T}_{\mathrm{scm}}\right)$, $\mathrm{T}$ central memory $\left(\mathrm{T}_{\mathrm{cm}}\right), \mathrm{T}$ effector memory $\left(\mathrm{T}_{\mathrm{em}}\right)$, and $\mathrm{T}$ effector memory CD45RA positive (TEMRA).

ity are from the $\mathrm{T}$ stem cell memory $\left(\mathrm{T}_{\mathrm{scm}}\right)$ compartment. Infusion of less differentiated memory $\mathrm{CD} 8^{+} \mathrm{T}$ cells in both mouse and non-human primate models have demonstrated increased engraftment and T-cell persistence [19-22]. Many ex vivo T-cell expansion systems involve processes that drive $\mathrm{T}$ cells toward a state of terminal differentiation and exhaustion. These $\mathrm{T}$ cells are known as T effector memory CD45RA-positive (TEMRA) cells. As a result, many of the cells in the final T-cell products using these systems maintain potency but are very shortlived. A primary driver of tumor relapse after initial response from these therapies is loss of circulating $\mathrm{T}$ cells due to the death of the short-lived TEMRA cells [10, 23, 24].

The AIM-np-based clinical manufacturing system consistently produces a $\mathrm{T}$-cell product comprised of $\sim 90 \% \mathrm{~T}_{\mathrm{scm}}, \mathrm{T}_{\mathrm{cm}}$, and $\mathrm{T}_{\mathrm{em}} \mathrm{CD} 8^{+} \mathrm{T}$ cells (shown in Fig. $4 \mathrm{~B}$ ). Noteworthy is that starting leukapheresis material contains a large population of naïve T cells on day $0(\sim 21 \%)$, while the final product contains on average less than $3 \%$ naïve $\mathrm{T}$ cells, as shown in Figure 4A. Furthermore, as demonstrated by Ichikawa et al. [18], similar memory cell populations and subtypes are also produced from relapsed refractory melanoma patients when compared to healthy donors. Thus, this AIM-np-based approach reproducibly manufactures $\mathrm{T}$-cell products that have the desired combination of memory phenotypes that have the potential to mediate an effective and long-lasting anti-tumor response.

\section{T-Cell Functionality}

Alongside phenotype and specificity, functionality is another important factor for successful T-cell therapy. Apart from tumor-specific cytotoxicity, release of immune-mediating cytokines is an important measure of effector function. The ability of $\mathrm{T}$ cells to release multiple cytokines in response to a pathogen is considered a marker of in vivo functionality. Furthermore, it has been shown that the ability to produce multiple cytokines is paramount to a robust $\mathrm{T}$ cell-mediated immune response and, in fact, serves as a correlate for T-cell efficacy $[25,26]$. We have assessed the polyfunctionality of our final T-cell products via intracellular cytokine staining flow cytometry, specifically looking at production of IL-2, IFN- $\gamma$, $\mathrm{TNFa}$, and an upregulation of CD107a, an indicator of cytolytic activity. On average, $60 \%$ of the T cells in our cellular products demonstrate 3-4 distinct effector functions, indicating a better in vivo therapeutic profile after infusion (shown in Fig. 3C, D).

\section{Clinical Applications}

Utilizing antigen-specific $\mathrm{CD} 8^{+} \mathrm{T}$ cells generated via our expansion platform, two multi-institutional phase $1 / 2$ clinical trials have been initiated. The company's lead indication will evaluate its NEXI-001 T-cell product in AML patients with relapsed disease after receiving an allogeneic stem cell transplant (ClinicalTrials.gov identifier: NCT04284228). Patients enrolled on this study will 
receive AML tumor-specific $\mathrm{CD} 8^{+} \mathrm{T}$ cells produced from the original HLA-matched stem cell donor peripheral blood mononuclear cells. The primary objectives for this trial are safety and tolerability. Six patients will be enrolled in two separate cohorts at escalating dose levels within a $3+3$ design. In absence of any dose-limiting toxicities, a dose expansion phase will follow, allowing for enrollment of up to 16-20 additional patients. All patients on study will be monitored for dose-limiting toxicities, adverse events, immunologic response, and clinical activity.

The company's second trial is currently enrolling relapsed/refractory multiple myeloma patients that have failed at least three prior lines of therapy (ClinicalTrials. gov identifier: NCT04505813). Similarly, the goal of this trial is to evaluate safety and tolerability. For this trial, each NEXI-002 T-cell product will be produced from individual patient-derived leukopak. Initial results from both trials were presented at the virtual 2020 ASH Conference.

\section{Conclusion}

In summary, the AIM-np technology is a customizable, "off the shelf" technology that serves as synthetic APCs to direct the natural immune function of antigenspecific $\mathrm{CD}^{+} \mathrm{T}$ cells. The AIM-np offers a controlled method for antigen presentation and T-cell co-stimula- tion by directly engaging antigen-specific $\mathrm{T}$ cells. When coupled with the E+E manufacturing system, the AIM-np provide antigen-specific T-cell stimulation and expansion through a highly controllable, closed, automated, and scalable process. The final products are consistent in composition, are comprised of $\mathrm{T}$ cells expanded from the endogenous repertoire and contain $\mathrm{T}$ cells with naturally occurring T-cell receptor affinities. These attributes have potential to greatly reduce the risk of the life-threatening toxicities associated with on-target/off-tissue activity observed with genetically engineered $\mathrm{T}$-cell products. In addition to purity of composition, AIM-expanded cellular products contain populations of $\mathrm{T}$ cells that are tumor antigen-specific, cytotoxic, and polyfunctional and of memory subtypes capable of generating potent and durable clinical responses. Finally, the AIM system is highly reproducible, scalable, and cost-effective, which increases the potential to access broader patient populations.

\section{Conflict of Interest Statement}

All listed authors are employees of NexImmune, Inc.

\section{Author Contributions}

L.S., R.W., M.O., D.B., H.M., K.J., and S.C. all contributed to the writing, review, and discussion of the manuscript.

\section{References}

1 Wölfl M, Greenberg PD. Antigen-specific activation and cytokine-facilitated expansion of naive, human CD8+ T cells. Nat Protoc. 2014 Apr;9(4):950-66.

2 Posch W, Lass-Flörl C, Wilflingseder D. Generation of Human Monocyte-derived Dendritic Cells from Whole Blood. JoVE. 2016;(118):e54968.

3 Gabrilovich D. Mechanisms and functional significance of tumour-induced dendritic-cell defects. Nat Rev Immunol. 2004 Dec;4(12): 941-52.

4 Sica A, Bronte V. Altered macrophage differentiation and immune dysfunction in tumor development. J Clin Invest. 2007 May;117(5): 1155-66.

5 Sotomayor EM, Borrello I, Rattis FM, Cuenca AG, Abrams J, Staveley-O'Carroll K, et al. Cross-presentation of tumor antigens by bone marrow-derived antigen-presenting cells is the dominant mechanism in the induction of T-cell tolerance during B-cell lymphoma progression. Blood. 2001 Aug;98(4): 1070-7.

6 Perica K, Bieler JG, Schütz C, Varela JC, Douglass J, Skora A, et al. Enrichment and Expansion with Nanoscale Artificial Antigen Presenting Cells for Adoptive Immunotherapy. ACS Nano. 2015 Jul;9(7):6861-71.
7 Lulla PD, Tzannou I, Vasileiou S, Carrum G, Ramos CA, Kamble R, et al. The safety and clinical effects of administering a multiantigen-targeted $\mathrm{T}$ cell therapy to patients with multiple myeloma. Sci Transl Med. 2020 Jul; 12(554):eaaz3339. https://doi.org/10.1126/ scitranslmed.aaz3339.

8 Shimizu J, Yamazaki S, Sakaguchi S. Induction of tumor immunity by removing CD25+CD4+ T cells: a common basis between tumor immunity and autoimmunity. J Immunol. 1999 Nov;163(10):5211-8.

9 Gattinoni L, KlebanoffCA, Restifo NP. Paths to stemness: building the ultimate antitumour $\mathrm{T}$ cell. Nat Rev Cancer. 2012 Oct;12(10):671-84.

10 Gattinoni L, Speiser DE, Lichterfeld M, Bonini C. T memory stem cells in health and disease. Nat Med. 2017 Jan;23(1):18-27.

11 Pollack SM, Jones RL, Farrar EA, Lai IP, Lee SM, Cao J, et al. Tetramer guided, cell sorter assisted production of clinical grade autologous NY-ESO-1 specific CD8(+) T cells. J Immunother Cancer. 2014 Oct;2(1):36.

12 Chapuis AG, Desmarais C, Emerson R, Schmitt TM, Shibuya K, Lai I, et al. Tracking the fate and origin of clinically relevant adoptively transferred CD8+ T cells in vivo. Sci Immunol. 2017 Feb;2(8):eaal2568. https://doi. org/10.1126/sciimmunol.aal2568.
13 Turtle CJ, Hudecek M, Jensen MC, Riddell SR. Engineered T cells for anti-cancer therapy. Curr Opin Immunol. 2012 Oct;24(5):6339.

14 Sotillo E, Barrett DM, Black KL, Bagashev A, Oldridge D, Wu G, et al. Convergence of Acquired Mutations and Alternative Splicing of CD19 Enables Resistance to CART-19 Immunotherapy. Cancer Discov. 2015 Dec;5(12): 1282-95.

15 Anguille S, Van Tendeloo VF, Berneman ZN. Leukemia-associated antigens and their relevance to the immunotherapy of acute myeloid leukemia. Leukemia. 2012 Oct;26(10):218696.

16 Ochsenreither S, Majeti R, Schmitt T, Stirewalt D, Keilholz U, Loeb KR, et al. Cyclin-A1 represents a new immunogenic targetable antigen expressed in acute myeloid leukemia stem cells with characteristics of a cancer-testis antigen. Blood. 2012 Jun;119(23):5492501.

17 Goswami M, Hensel N, Smith BD, Prince GT, Qin L, Levitsky HI, et al. Expression of putative targets of immunotherapy in acute myeloid leukemia and healthy tissues. Leukemia. 2014 May;28(5):1167-70. 
18 Ichikawa J, Yoshida T, Isser A, Laino AS, Vassallo M, Woods D, et al. Rapid Expansion of Highly Functional Antigen-Specific T Cells from Patients with Melanoma by Nanoscale Artificial Antigen-Presenting Cells. Clin Cancer Res. 2020 Jul;26(13):3384-96.

19 Gattinoni L, Lugli E, Ji Y, Pos Z, Paulos CM, Quigley MF, et al. A human memory T cell subset with stem cell-like properties. Nat Med. 2011 Sep;17(10):1290-7.

20 Berger C, Jensen MC, Lansdorp PM, Gough M, Elliott C, Riddell SR. Adoptive transfer of effector CD8+ T cells derived from central memory cells establishes persistent $\mathrm{T}$ cell memory in primates. J Clin Invest. 2008 Jan; 118(1):294-305.
21 Klebanoff CA, Gattinoni L, Torabi-Parizi P, Kerstann K, Cardones AR, Finkelstein SE, et al. Central memory self/tumor-reactive CD8+ $\mathrm{T}$ cells confer superior antitumor immunity compared with effector memory T cells. Proc Natl Acad Sci USA. 2005 Jul;102(27):9571-6.

22 Wang X, Wong CW, Urak R, Taus E, Aguilar $\mathrm{B}$, Chang WC, et al. Comparison of naïve and central memory derived CD8+ effector cell engraftment fitness and function following adoptive transfer. OncoImmunology. 2015 Aug;5(1):e1072671.

23 Bleakley M, Heimfeld S, Jones LA, Turtle C, Krause D, Riddell SR, et al. Engineering human peripheral blood stem cell grafts that are depleted of naïve $\mathrm{T}$ cells and retain functional pathogen-specific memory T cells. Biol Blood Marrow Transplant. 2014 May;20(5):705-16.
24 Triplett BM, Shook DR, Eldridge P, Li Y, Kang G, Dallas M, et al. Rapid memory T-cell reconstitution recapitulating CD45RA-depleted haploidentical transplant graft content in patients with hematologic malignancies. Bone Marrow Transplant. 2015 Jul;50(7): 968-77.

25 Boyd A, Almeida JR, Darrah PA, Sauce D, Seder RA, Appay V, et al. Pathogen-Specific T Cell Polyfunctionality Is a Correlate of T Cell Efficacy and Immune Protection. PLoS One. 2015 Jun;10(6):e0128714.

26 Han Q, Bagheri N, Bradshaw EM, Hafler DA, Lauffenburger DA, Love JC. Polyfunctional responses by human $\mathrm{T}$ cells result from sequential release of cytokines. Proc Natl Acad Sci USA. 2012 Jan;109(5):1607-12. 